\title{
Application of a Vis-NIR Spectroscopic Technique to Measure the Total Soluble Solids Content of Intact Mangoes in Motion on a Belt Conveyor
}

\author{
Sneha Sharma ${ }^{1}$, Panmanas Sirisomboon ${ }^{1 *}$ and Pimpen Pornchaloempong ${ }^{2}$ \\ ${ }^{1}$ Department of Agricultural Engineering, Faculty of Engineering, King Mongkut's Institute of Technology Ladkrabang, 1 \\ Chalongkrung Road, Ladkrabang, Bangkok 10520, Thailand \\ ${ }^{2}$ Department of Food Engineering, Faculty of Engineering, King Mongkut's Institute of Technology Ladkrabang, 1 Chalongkrung \\ Road, Ladkrabang, Bangkok 10520, Thailand
}

To date, different approaches have been applied to measure the internal parameters of mangoes by destructive and non-destructive techniques. Recently, real-time evaluation of the internal parameters has become important assessment for fruits in high demand. This research aims to develop an online prototype system to measure the total soluble solids (TSS) in mangoes using a fiber optic diode array Visible-Near Infrared (VisNIR) spectrometer on a conveyor belt. Spectra were acquired in a wavelength range from $400-1000 \mathrm{~nm}$. The diffuse reflectance spectra of mangoes were subjected to several preprocessing techniques such as moving average smoothing (MAS), standard normal variate (SNV), multiplicative scatter correction (MSC), baseline offset and normalization before model development. The spectral information and corresponding TSS values were used to establish a linear relationship by partial least squares (PLS) regression. Spectra in three wavelength ranges of $400-1000 \mathrm{~nm}, 600-1000 \mathrm{~nm}$, and $700-1000 \mathrm{~nm}$ were used for the model development. Baseline offset combined with MAS showed effective transformation of spectra at a wavelength of 600$1000 \mathrm{~nm}$. The optimum model was obtained by an external validation technique with a correlation coefficient of calibration set and a prediction set of 0.80 and 0.74 , respectively. The root mean square error of the calibration (RMSEC), root mean square error of prediction (RMSEP) and bias were $0.690 \%, 0.765 \%$, and $0.061 \%$, respectively. The statistical results from PLS regression indicated the feasibility of using the online conveyor system for grading the fruit according to the TSS.

Key Words: mango, near Infrared (NIR) spectroscopy, online conveying system, total soluble solids.

\section{Introduction}

Statistics for January to December 2017 and 2018 show that approximately $30,783,364 \mathrm{~kg}$ of fresh mangoes worth around 1.05 million USD were exported to several countries from Thailand (Thai customs, $<$ http://www.customs.go.th/statistic_report.php?lang= en\&show_search $=1>$, September, 2018). This figure shows the demand for the fruit worldwide and also suggests the impact of the fruit export rate on the country's economy. Mango is a popular fruit because of its taste and attractive appearance, so to maintain the demand and supply levels of this fruit, the quality during the exporting process has to be determined in

Received; November 25, 2019. Accepted; May 11, 2020.

First Published Online in J-STAGE on July 14, 2020.

No conflicts of interest declared.

* Corresponding author (E-mail: panmanas.si@kmitl.ac.th). the most efficient manner.

The quality attributes of mangoes can be measured by the dry matter content (DM), firmness, fruit color (both peel and flesh), total soluble solids (TSS), titratable acidity (TA), and aromatic compounds (Padda et al., 2011; Sivakumar et al., 2011; Subedi et al., 2007; Ueda et al., 2000). During the ripening stage, there is an increase in the soluble sugar content in mangoes which is usually expressed as TSS as most of the solids are sugar (Schmilovitch et al., 2000). The total soluble solids content, and to a small extent sucrose, glucose, and fructose, are the typical quality attributes used to assess the sweetness of mangoes and have demonstrated their usefulness in determining the physicochemical changes during the ripening stages (Delwiche et al., 2008). The sugars were identified as glucose, fructose, and sucrose in four mango cultivars by Liu et al. (2013), among which sucrose was the predominant sugar. Total soluble solids concentration is represented 
in TSS $\%$ or ${ }^{\circ}$ Brix. Jha et al. (2006) reported that TSS in mangoes increases from 6.9-8.1 ${ }^{\circ}$ Brix until reaching the maturity stage and gradually increases from 8-13 ${ }^{\circ}$ Brix after attaining maturity. The TSS content of stored fruit increases up to $19^{\circ}$ Brix. Analysis of the internal quality of mangoes has been done by both destructive and nondestructive techniques (Marques et al., 2016; Ngamchuachit et al., 2015). Destructive methods are not feasible for the continuous determination of the internal quality of exported fruits. Therefore, rapid nondestructive quality analysis of fruit in processing sites is in high demand nowadays. Much research has shown the potential of using the NIR spectroscopic technique to measure different internal parameters of fruits. This technique uses the NIR range of electromagnetic radiation to measure different parameters of the analytes. As the NIR radiation interacts with the analytes or sample, radiation photons interact with the material within the analytes at the molecular level (Slaughter, 2009). According to the wavelength, the radiation is characterized as visible light with a wavelength range in the $400 \mathrm{~nm}$ to $700 \mathrm{~nm}$ region, while the near-infrared region has wavelengths between $700 \mathrm{~nm}$ and $2500 \mathrm{~nm}$ (Wang and Paliwal, 2007). When the radiation comes into contact with a molecule, it can either be absorbed, reflected from or transmitted through the molecule. Thus, the wavelength of the radiation absorbed, reflected or transmitted by the molecule indicates the type of molecule, which can be water, sugar, starch, protein, fat, etc., in the analytes.

Most NIR spectroscopic applications have been done under offline conditions, but research to identify the potential of online application to analyze the quality parameters of agricultural products is increasing. Using diode array spectrometers, the online analysis of several parameters in fruits and vegetables is possible. Tian et al. (2007) indicated the feasibility of Vis/NIR diffuse transmittance spectral analysis to predict watermelon SSC in a nondestructive way. Jie et al. (2014) used the Vis-NIR diffuse transmittance technique to predict the soluble solids content of watermelons in an online detection system. Phetpan et al. (2018) developed a prototype system for online SSC prediction in sugarcane using visible and near-infrared spectroscopy (Vis/SWNIR). The results obtained by scanning sugarcane billets online on a conveyor system were good and showed the potential of using a diode array spectrometer to develop a robust regression model. SalgueroChaparro et al. (2013) reported the feasibility of online scanning using a diode array NIR spectrometer for intact olive fruits. This research showed good evidence using an online scanning condition with the NIR spectroscopic technique. Also, Cortés et al. (2019) evaluated a new non-destructive in-line Vis-NIR spectroscopy prototype for in-line identification of five apple varieties. The results from Cortés et al. (2019) indicated that the in-line application of Vis-NIR spectroscopy for variety classification is feasible with an accuracy that is similar to, or better than, a laboratory or at-line system. For a fruit such as mango, it is now neccesary to develop a similar technique for rapid and accurate quality analysis. Experimental studies showed that NIR diffuse reflectance measurements can obtain valuable information about the chemical and physical properties of several fruits, both in motion and statically ( $\mathrm{Lu}$ et al., 2000; Salguero-Chaparro et al., 2012). Therefore, this research aims to determine the feasibility of online diffuse reflectance scanning using a diode array short wave Vis-NIR spectrometer to measure TSS in mangoes moving along a belt conveyor.

\section{Materials and Methods}

\section{Samples}

The mango cultivar Nam Dok Mai Sithong was obtained from an orchard located in Chacheangsao province in Thailand. A total number of 182 samples were used. Mango fruit of different sizes were collected, and the dimensional information is summarized in Table 1. All the samples ready for commercial export were selected randomly for this experiment. Samples were kept at room temperature at $25 \pm 1{ }^{\circ} \mathrm{C}$ for 2 hours before scanning.

\section{Online conveying system and spectral acquisition}

The online conveying system developed in this study consists of four main parts, as shown in Figure 1: a) an infrared motion sensor to detect the presence and movement of the samples; b) a Vis-NIR AvaSpec-2048USB2 standard fiber optic spectrometer (Avantes, the Netherlands), wavelength range of $350-1160 \mathrm{~nm}$ with a charged coupled device (CCD) detector array; c) an

Table 1. Summary of sample dimensions.

\begin{tabular}{ccccc}
\hline \hline $\begin{array}{c}\text { No of } \\
\text { samples }\end{array}$ & Dimension & $\begin{array}{c}\text { Min } \\
(\mathrm{mm})\end{array}$ & $\begin{array}{c}\text { Max } \\
(\mathrm{mm})\end{array}$ & $\begin{array}{c}\text { Average } \\
(\mathrm{mm})\end{array}$ \\
\hline \multirow{3}{*}{182} & Length & 135 & 158 & 143 \\
& Width & 74 & 95 & 84 \\
& Depth & 66 & 75 & 71 \\
\hline
\end{tabular}

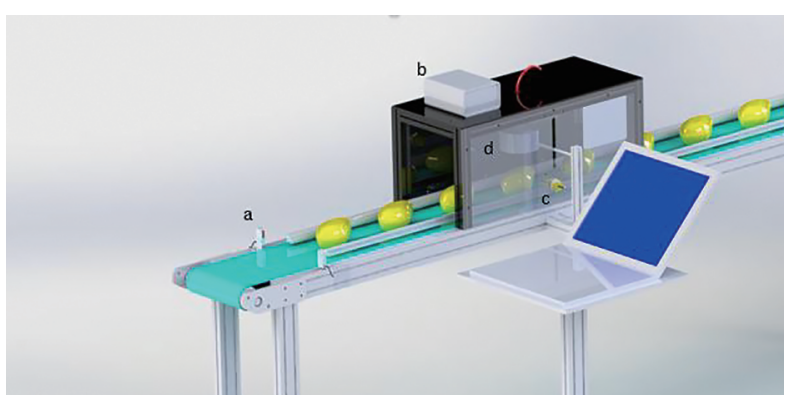

Fig. 1. Online scanning system for measuring the TSS of mangoes using a Vis-NIR Spectrometer: (a) Infrared motion sensor, (b) Vis-NIR Spectrometer, (c) Proximity sensor, (d) Integrating sphere. 
integrating sphere (Avasphere 50-REFL; Avantes) probe equipped with a halogen light source mounted on the top of a black box ( $60 \mathrm{~cm}$ long); d) a proximity sensor as the external trigger to the spectrometer. Metal rods were attached to the conveyor to guide the sample towards the spectrometer. The speed of the conveyor belt was fixed at $0.1 \mathrm{~m} \cdot \mathrm{s}^{-1}$. Each sample was passed through the black box fixed on the conveyor, thus eliminating interference from external light. The proximity sensor was fixed to the side of the black box such that the integrating sphere and the proximity sensor were aligned on the same vertical plane.

A light source connected to the spectrometer was allowed to warm up for 15-30 minutes before scanning. A reference and dark spectrum was acquired to correct the disturbance in the light source intensity and background noise within the detector. The reference spectrum was captured using a white Teflon material. The integration time, i.e., the length of time that the detector could read the radiation, was adjusted so the maximum count over the wavelength range was around $90 \%$ of the full analog to digital converter (ADC) scale of reflectance from the reference material. The optimum integration time of $3.7 \mathrm{~ms}$ was set with a focal length between the sample and the sensor of approximately $2.5 \mathrm{~cm}$. However, the focal length changes with the size of the sample. As the sample approaches the proximity sensor, it triggers the spectrometer to start scanning along the fruit's surface. An average of 200 spectra were obtained after scanning each sample.

\section{Reference laboratory test to measure TSS}

After spectral acquisition, the samples were used for reference lab analysis i.e. TSS measurement. Each sample was sliced longitudinally and separated into three parts: the shoulder, cheek, and tip. The pulp was squeezed directly into a Digital Hand-held "Pocket" Refractometer (PAL-1 S/No L218454; Atago, Japan). Distilled water was used as the standardizing liquid for the refractometer. Three repeat measurements of TSS were done for each part, that is, the shoulder (the uppermost part attached to the stem), the cheek (the middle part), and the tip (the lowermost part), as shown in Figure 2, and the average TSS measured from each part was recorded as the reference TSS value.

\section{Data analysis}

The wavelength ranges of $350-400 \mathrm{~nm}$ and over $1000 \mathrm{~nm}$ were removed due to excessive noise. The Unscrambler X version 10.5.1 (Camo, Norway) was used for the preprocessing and model development using different wavelength ranges. After spectral acquisition, the total number of spectra (182) acquired was divided into calibration and validation sets. A total of 182 samples were used in the experiments. The division of all samples used was done at a ratio of 80:20 of the total sample size. Therefore, from the acquired spectral

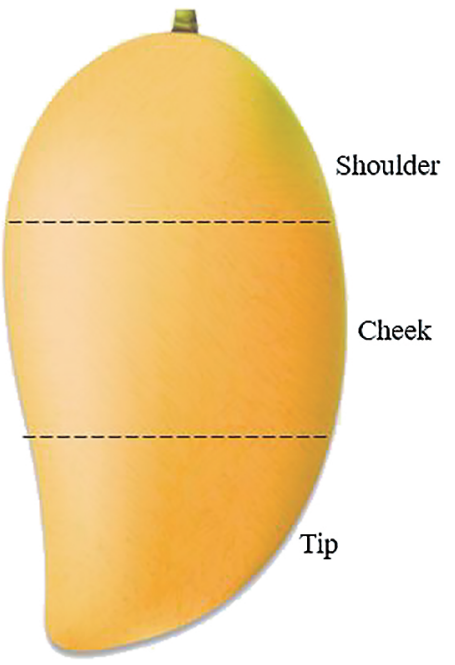

Fig. 2. Division of whole fruit into three parts.

set, 146 samples for the calibration set and 36 for the validation set were separated randomly. An external validation method was used to develop the model with a calibration spectra set of 146, and 36 spectra to validate the model developed by the calibration set.

Moving average smoothing (MAS) with 11 segments following several pretreatments such as standard normal variate (SNV), multiplicative scatter correction (MSC), baseline offset, and normalization were applied to the raw spectra. SNV and MSC transformation are usually applied to spectra to ;remove scatter effects by centering and scaling each spectrum. Baseline offset correction was used to adjust the spectral offset by adjusting the data to the minimum point (Camo Software, 2006; <https://www.camo.com/downloads/U9.6\%20pdf $\% 20$ manual/The \%20Unscrambler\%20Methods.pdf $>$.). Equation (1) gives the formula for the baseline offset correction

$$
f(x)=x-\min X
$$

where, $x$ is the absorbance value of a sample at a particular wavelength and $X$ denotes all absorbance values of the sample (Camo Software, 2006). For each sample, the value of the lowest point in the spectrum was subtracted from all the variables. Normalization reduces unwanted sources of variability by linking a group of spectra with more common features. A normalization scale was used for the samples' spectra to get all data on approximately the same scale (Camo software, 2006). Equation (2) gives the transformation formula for normalization where each observation in the data matrix $\left(\mathrm{X}_{\mathrm{i}, \mathrm{k}}\right)$ is divided by its average value $\overline{\mathrm{X}}$ (Conzen, 2006)

$$
\mathrm{X}_{\mathrm{i}, \mathrm{k}} \frac{\mathrm{X}_{\mathrm{i}, \mathrm{k}}}{|\overline{\mathrm{X}}|}
$$

Three wavelength ranges were selected for the model optimization. These were $400-1000 \mathrm{~nm}$ (Vis-NIR), 600-1000 nm (Vis-NIR) and 700-1000 nm (NIR). The 


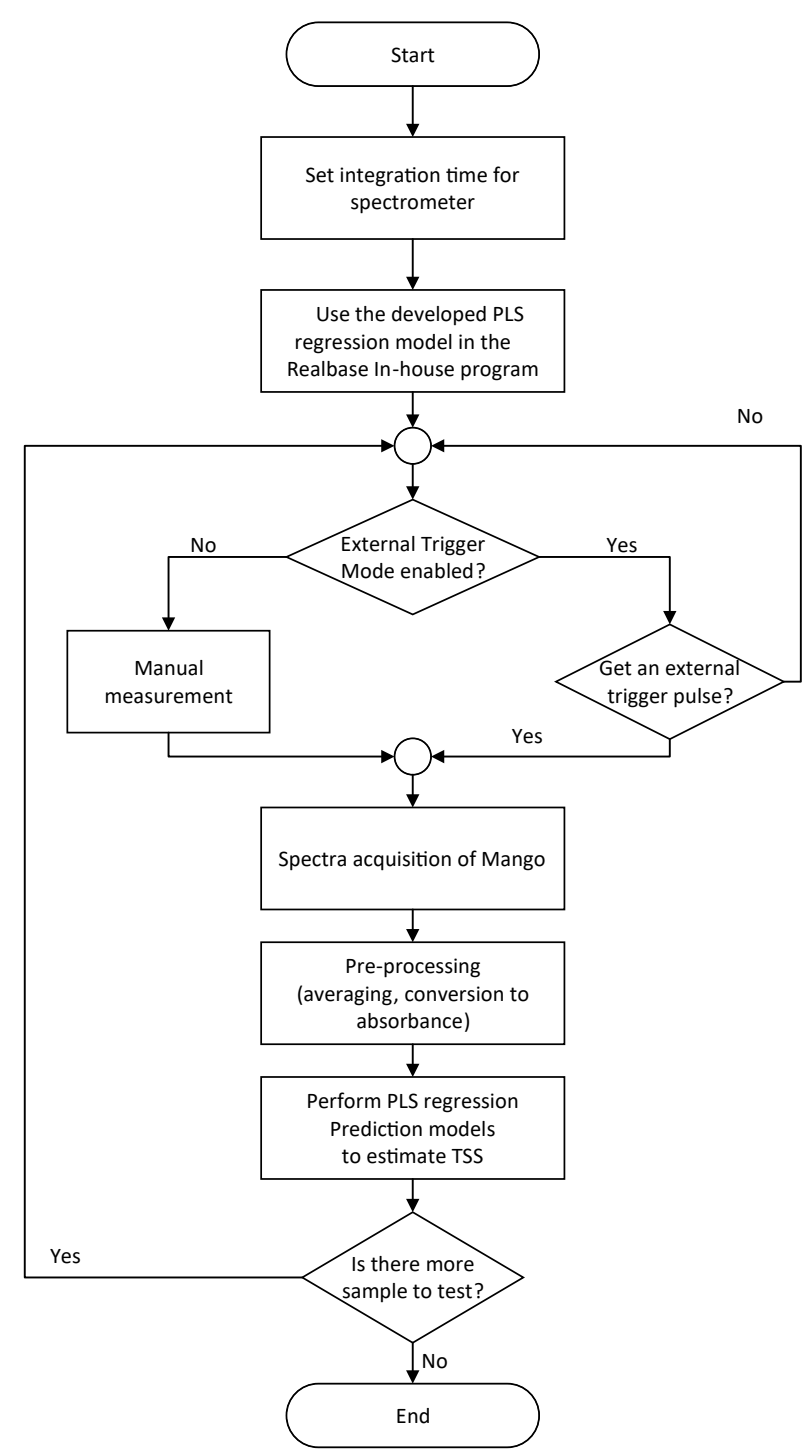

Fig. 3. Flowchart showing the Realbase program developed inhouse.

optimum wavelength range was selected from the best statistics, which were compared based on the correlation coefficient (R), the root mean square error of calibration (RMSEC), root mean square error of prediction (RMSEP), and bias of the model developed using partial least squares regression (PLSR).

After obtaining the optimum wavelength and regression model, the model was integrated into an online system. The Realbase In-house program was developed using the Python programming language. This program works as an interface between the spectrometer and the computer to obtain spectra, model evaluation and TSS prediction. The program can be used with online conveying system in the packaging house or firm for real time TSS prediction of mangoes. A working flowchart of the program developed is shown in Figure 3.

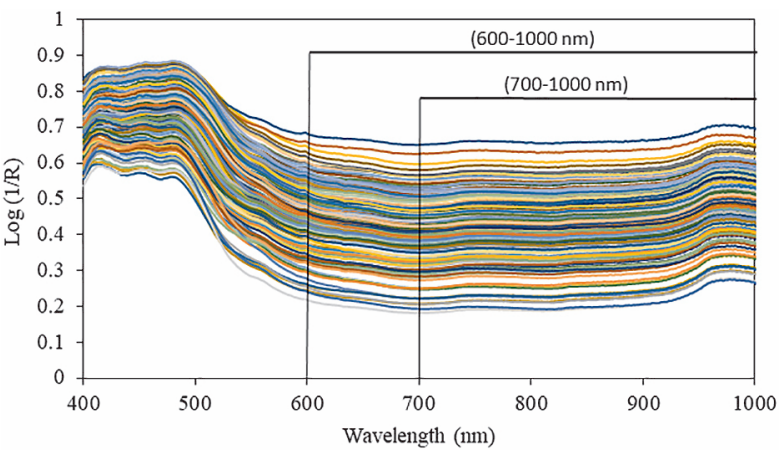

Fig. 4. Raw spectra obtained by online scanning of mangoes using a Vis/NIR spectrometer.

\section{Results and Discussion}

Spectral characteristics

To achieve consistency in any spectra obtained, several parameters were considered. To optimize the parameters, such as integration time and average focal distance, the repeatability and reproducibility were calculated before the experiment. The raw spectra obtained from the three wavelength ranges of $400-1000 \mathrm{~nm}$, $600-1000 \mathrm{~nm}$, and $700-1000 \mathrm{~nm}$ are shown in Figure 4. In the raw spectra, a relatively high absorption peak was observed in the visible range compared to the NIR region. Likewise, the spectra were affected by noise and the scattering effect due to the change in the focal length between the samples. Some pre-processing techniques were applied to the raw spectra to minimize errors. Figure 5 shows the spectra after applying a combination of pretreatment in the wavelength range of 600-1000 nm. Two small peaks appeared around $760 \mathrm{~nm}$ and $970 \mathrm{~nm}$, which are the absorption bands of water.

The main motive for using preprocessing techniques is to improve the spectral characteristics by reducing scattering effects caused by physical variability between samples and correcting the baseline shift. SNV, MSC, normalization and baseline shift are well known to reduce such errors caused by scattering and improve the spectra (as seen in Figs. 4 and 5). The signal to noise ratio can be improved by applying the moving average smoothing technique. The spectra obtained using different combinations of pretreatments gave approximately the same characteristics as shown in Figure 5. The statistical data of the TSS measured to develop the PLS regression model are shown in Table 2. Tables 3, 4, and 5 show the PLS regression models of three wavelength ranges.

\section{PLS regression model selection}

From the results obtained from the PLS regression by applying pretreatment to different wavelength ranges, the optimum wavelength range and a regression model were selected. Comparing the results shown in Tables 3, 4 , and 5 based on statistical parameters, the results from 

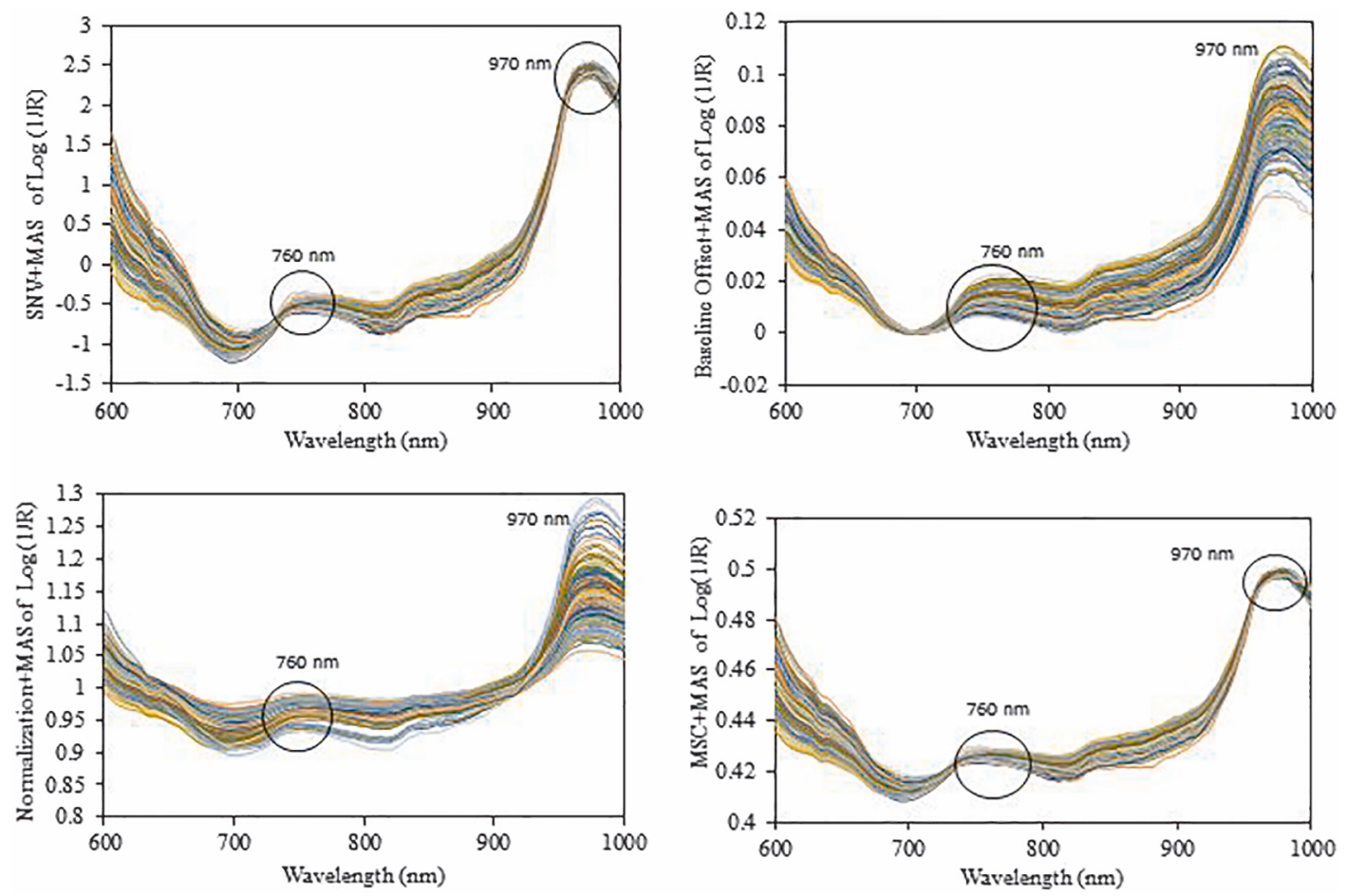

Fig. 5. Pretreated spectra with pretreatment SNV, baseline offset, normalization, and MSC along with moving average smoothing (MAS) in a wavelength range from $600-1000 \mathrm{~nm}$.

Table 2. Statistical data of TSS measured for model development.

\begin{tabular}{|c|c|c|c|c|c|c|c|c|c|c|}
\hline \multirow{2}{*}{ Parameter } & \multicolumn{5}{|c|}{ Calibration set } & \multicolumn{5}{|c|}{ Validation set } \\
\hline & $\mathrm{n}$ & Mean & $\operatorname{Max}$ & Min & SD & $\mathrm{n}$ & Mean & Max & Min & SD \\
\hline TSS (\%) & 146 & 17.0 & 19.8 & 14.1 & 1.2 & 36 & 17.1 & 19.6 & 15.2 & 1.1 \\
\hline
\end{tabular}

$\mathrm{n}=$ Number of samples, Max $=$ Maximum TSS value, Min $=$ Minimum TSS value, $\mathrm{SD}=$ Standard Deviation.

Table 3. PLS regression model obtained for TSS prediction using a wavelength range of $400-1000 \mathrm{~nm}$.

\begin{tabular}{|c|c|c|c|c|c|c|}
\hline \multirow{2}{*}{ Preprocessing } & \multirow{2}{*}{ Factor } & \multicolumn{2}{|c|}{ Calibration set } & \multicolumn{3}{|c|}{ Validation set } \\
\hline & & $R$ & RMSEC (\%) & $r$ & RMSEP (\%) & Bias \% \\
\hline Raw & 7 & 0.74 & 0.786 & 0.57 & 0.942 & -0.002 \\
\hline $\mathrm{SNV}+\mathrm{MAS}$ & 7 & 0.76 & 0.755 & 0.69 & 0.821 & -0.006 \\
\hline Baseline Offset + MAS & 7 & 0.72 & 0.809 & 0.53 & 0.965 & 0.023 \\
\hline $\mathrm{MSC}+\mathrm{MAS}$ & 7 & 0.76 & 0.755 & 0.69 & 0.821 & -0.007 \\
\hline Normalization +MAS & 7 & 0.72 & 0.804 & 0.56 & 0.952 & -0.005 \\
\hline
\end{tabular}

$\mathrm{SNV}=$ Standard normal variate, $\mathrm{MSC}=$ Multiplicative scatter correction, $\mathrm{MAS}=$ Moving average smoothing .

Table 4. PLS regression model obtained for TSS prediction using a wavelength range of 600-1000 nm.

\begin{tabular}{|c|c|c|c|c|c|c|}
\hline \multirow{2}{*}{ Preprocessing } & \multirow{2}{*}{ Factor } & \multicolumn{2}{|c|}{ Calibration set } & \multicolumn{3}{|c|}{ Validation set } \\
\hline & & $R$ & RMSEC \% & $r$ & RMSEP \% & Bias \% \\
\hline Raw & 7 & 0.85 & 0.611 & 0.68 & 0.835 & 0.072 \\
\hline $\mathrm{SNV}+\mathrm{MAS}$ & 7 & 0.80 & 0.693 & 0.72 & 0.793 & 0.061 \\
\hline Baseline Offset + MAS & 7 & 0.80 & 0.690 & 0.74 & 0.765 & 0.061 \\
\hline $\mathrm{MSC}+\mathrm{MAS}$ & 7 & 0.82 & 0.656 & 0.68 & 0.835 & 0.034 \\
\hline Normalization + MAS & 7 & 0.79 & 0.711 & 0.72 & 0.800 & 0.082 \\
\hline
\end{tabular}

$\mathrm{SNV}=$ Standard normal variate, $\mathrm{MSC}=$ Multiplicative scatter correction, $\mathrm{MAS}=$ Moving average smoothing. 
Table 5. PLS regression model obtained for TSS prediction using a wavelength range of 700-1000 nm.

\begin{tabular}{|c|c|c|c|c|c|c|}
\hline \multirow{2}{*}{ Preprocessing } & \multirow{2}{*}{ Factor } & \multicolumn{2}{|c|}{ Calibration set } & \multicolumn{3}{|c|}{ Validation set } \\
\hline & & $R$ & RMSEC (\%) & $r$ & RMSEP (\%) & Bias \% \\
\hline Raw & 7 & 0.83 & 0.642 & 0.54 & 0.964 & 0.074 \\
\hline $\mathrm{SNV}+\mathrm{MAS}$ & 7 & 0.81 & 0.674 & 0.65 & 0.881 & 0.143 \\
\hline Baseline Offset+MAS & 7 & 0.79 & 0.709 & 0.58 & 0.953 & 0.168 \\
\hline $\mathrm{MSC}+\mathrm{MAS}$ & 7 & 0.81 & 0.676 & 0.65 & 0.881 & 0.146 \\
\hline Normalization + MAS & 7 & 0.80 & 0.695 & 0.58 & 1.038 & 0.140 \\
\hline
\end{tabular}

$\mathrm{SNV}=$ Standard normal variate, $\mathrm{MSC}=$ Multiplicative scatter correction, $\mathrm{MAS}=$ Moving average smoothing .

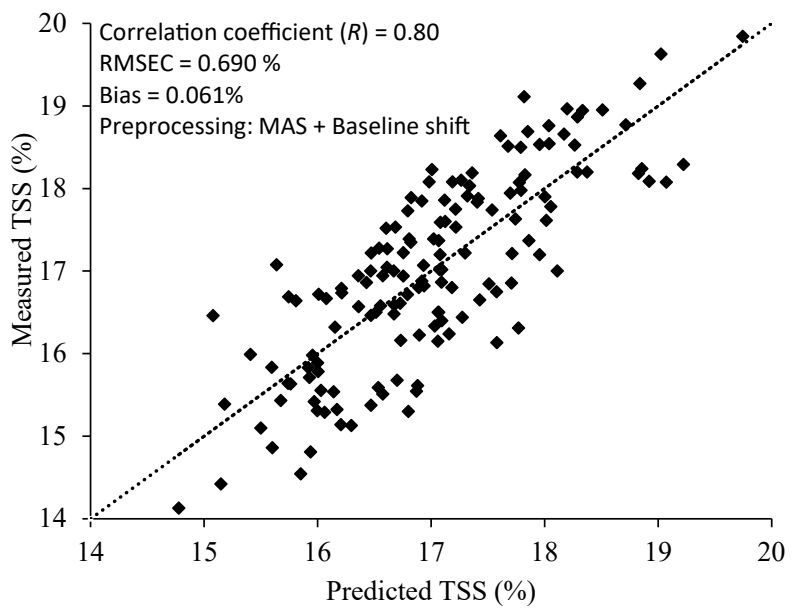

Fig. 6. Scatter plot of predicted TSS by online scanning versus measured TSS by reference laboratory analysis of the calibration data set.

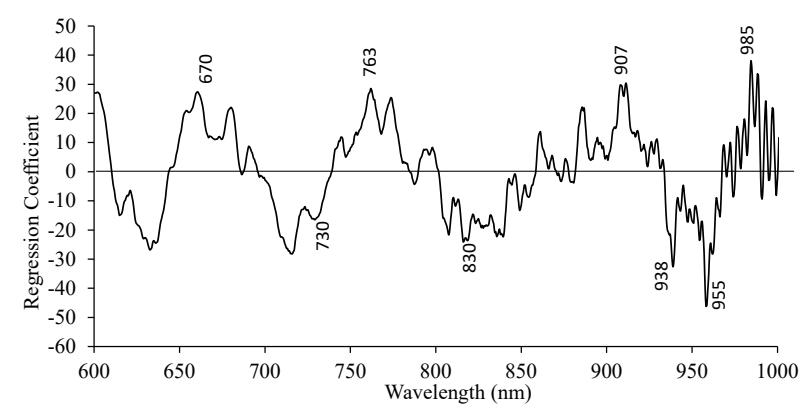

Fig. 7. Regression coefficient plot of the optimal model for measuring TSS in mangoes using an online scanning condition. the regression model developed on an NIR wavelength range of $700-1000 \mathrm{~nm}$ were the least accurate among the three ranges selected. In the NIR range (700$1000 \mathrm{~nm}$ ), the vibrations of the mango sample molecules were in the fourth and third overtone. These vibrations were generated only by the NIR absorbance which in the case of intact mangoes were not enough to predict TSS. The TSS prediction required more information from the visible range. The models developed at a wavelength range of $400-1000 \mathrm{~nm}$ and a range of $400-700 \mathrm{~nm}$ (full range of visible light) and 600 $1000 \mathrm{~nm}$, where 600-700 $\mathrm{nm}$ was the visible red region, gave better results, but comparing the RMSEC and RMSEP values, they were higher at the range of 400 $1000 \mathrm{~nm}$ than at $600-1000 \mathrm{~nm}$. As a result, the wavelength range of $600-1000 \mathrm{~nm}$ was selected as the optimum range for model development. The pre-processing techniques such as baseline offset, MSC and SNV combined with moving average smoothing provided almost the same results as these methods are based on the same mathematical concept. The best PLS regression model with the highest correlation coefficient and lowest root mean square error was obtained by applying baseline offset with moving average smoothing pre-processing. The correlation coefficient of calibration (R) was found to be 0.80 with a RMSEC of $0.690 \%$.

Figure 6 shows the correlation plot of predicted TSS by online scanning versus the measured TSS by the standard laboratory method. Figure 7 shows the regression coefficient plot obtained from the optimum model selected using PLS regression. The higher positive or negative peaks in the regression coefficient plot indi-

Table 6. Vibration bands of the peaks appearing in the regression coefficient plot.

\begin{tabular}{ccll}
\hline \hline Wavelength $(\mathrm{nm})$ & $\begin{array}{c}\text { Wavelength (nm) } \\
\text { Referred from reference }\end{array}$ & \multicolumn{1}{c}{ Bond vibration } & Structure \\
\hline 670 & 680 & & Chlorophyll* $^{*}$ \\
730 & 740 & O-H str. +6Third Overtone & Sucrose** $^{* *}$ \\
763 & 770 & O-H str. Third Overtone & Sucrose $^{* *}$ \\
830 & 840 & O-H Combination & Sucrose $^{* *}$ \\
907 & 910 & C-H str. Fourth Overtone & Sucrose** \\
955 & 960 & O-H str. Second Overtone & Sucrose** \\
985 & 984 & O-H str. Second Overtone & Sucrose** \\
\hline
\end{tabular}

* Abott et al. (1997) and **Golic et al. (2003). 
cate the influence of the vibration at the respective wavelength on the TSS prediction. In Table 6 , the vibration bands of the higher peaks observed in the regression plot are listed. Most of the highest peaks that appeared were due to the $\mathrm{O}-\mathrm{H}$ and $\mathrm{C}-\mathrm{H}$ combination or the overtone of the sucrose in water. These peaks have a direct influence on the TSS prediction. The peak of skin chlorophyll was observed at $680 \mathrm{~nm}$, which is one of the common peaks seen in the spectra of most fruits (Seifert et al., 2014). Soluble sugars such as glucose, fructose, and sucrose are the major soluble solids in fruits (Magwaza and Opara, 2015). The absorption bands relating to sugar components are considered important for TSS prediction. Therefore, from the regression coefficient plot, high upward and downward peaks at around $770 \mathrm{~nm}, 910 \mathrm{~nm}$, and $960 \mathrm{~nm}$ can be considered to be the most influential wavelengths for TSS prediction, and they also indicate the reliability of the regression model obtained.

RMSEP and RMSEC are considered to be important statistical parameters for regression model selection. According to previous studies, the lowest RMSEC obtained was $0.60{ }^{\circ}$ Brix and RMSEP was $0.89^{\circ}$ Brix for the TSS content in mangoes (Nordey et al., 2017). Considering several reports, it was concluded that the RMSEP can vary from $0.45{ }^{\circ}$ Brix to $1.55{ }^{\circ}$ Brix in mangoes (Delwiche et al., 2008; Marques et al., 2016; Rungpichayapichet et al., 2016; Schmilovitch et al., 2000 ), indicating that the RMSEP of $0.765 \%$ obtained in this study was comparable. A nondestructive online detection prototype system using Vis-NIR spectroscopy to predict the SSC of watermelon was performed by Jie et al. (2014) and the best prediction model was obtained with a correlation coefficient $(r)$ of 0.70 . The result showed that the online Vis-NIR spectroscopic technique for measuring the TSS of mangoes is feasible.

\section{Conclusion}

A prototype for an online scanning system to measure the TSS of mangoes using a non-destructive spectroscopic technique was developed. The speed of the conveyor was kept constant $\left(0.1 \mathrm{~m} \cdot \mathrm{s}^{-1}\right)$ and spectra were collected with an integration time of $3.7 \mathrm{~ms}$. The wavelength range used for scanning the samples was 400-1000 nm. Different wavelength ranges were used to select the best model. An optimum partial least squares regression (PLSR) model with a high correlation $(\mathrm{R})$ of 0.80 and the lowest RMSEC of $0.690 \%$ was obtained with a wavelength range of $600-1000 \mathrm{~nm}$. Further research in this field will include optimizing the model, as well as the system, at various speeds. A more robust model is expected by adding more samples to increase the TSS range and optimizing the conveying system based on different focal lengths and integration times at various conveyor speeds.

\section{Acknowledgements}

The authors would like to acknowledge King Mongkut's Institute of Technology Ladkrabang and the Thailand Government Budget Grant, Grant number 0161-007, for providing financial support and equipment to conduct this research. Also, the authors would like to thank the NIRS Research Center for Agricultural Product and Food (www.nirsresearch.com) at King Mongkut's Institute of Technology Ladkrabang, Bangkok, Thailand, for laboratory space and instruments used in for the experiment.

\section{Literature Cited}

Abbott, J. A., R. Lu, B. L. Upchurch and R. L. Stroshine. 1997. Technologies for non-destructive quality evaluation of fruits and vegetables. Hortic. Rev. 20: 1-120.

Conzen, J. P. 2006. Multivariate Calibration: A practical guide for developing methods in the quantitative analytical chemistry. $2^{\text {nd }}$ ed. Bruker Optik GmbH. Germany.

Cortés, V., S. Cubero, J. Blasco, N. Aleixos and P. Talens. 2019. In-line application of visible and near-infrared diffuse reflectance spectroscopy to identify apple varieties. Food Bioprocess Tech. 12: 1021-1030.

Delwiche, S. R., W. Mekwatanakarn and C. Y. Wang. 2008. Soluble solids and simple sugars measurement in intact mango using near infrared spectroscopy. HortTechnology 18: 410 416.

Golic, M., K. Walsh and P. Lawson. 2003. Short-wavelength near-infrared spectra of sucrose, glucose, and fructose with respect to sugar concentration and temperature. Appl. Spectrosc. 57: 139-145.

Jha, S. N., A. R. P. Kingsly and S. Chopra. 2006. Physical and mechanical properties of mango during growth and storage for determination of maturity. J. Food Eng. 72: 73-76.

Jie, D., L. Xie, X. Rao and Y. Ying. 2014. Using visible and near infrared diffuse transmittance technique to predict soluble solids content of watermelon in an online detection system. Postharvest Biol. Technol. 90: 1-6.

Liu, F.-X., S.-F. Fu, X.-F. Bi, F. Chen, X.-J. Liao, X.-S. Hu and J.-H. Wu. 2013. Physico-chemical and antioxidant properties of four mango (Mangifera indica L.) cultivars in China. Food Chem. 138: 396-405.

Lu, R., D. E. Guyer and R. M. Beaudry. 2000. Determination of firmness and sugar content of apples using near-infrared diffuse reflectance. J. Texture Stud. 31: 615-630.

Magwaza, L. S. and U. L. Opara. 2015. Analytical methods for determination of sugars and sweetness of horticultural products-A review. Sci. Hortic. 184: 179-192.

Marques, E. J. N., S. T. de Freitas, M. F. Pimentel and C. Pasquini. 2016. Rapid and non-destructive determination of quality parameters in the "Tommy Atkins" mango using a novel handheld near infrared spectrometer. Food Chem. 197: $1207-1214$.

Ngamchuachit, P., H. K. Sivertsen, E. J. Mitcham and D. M. Barrett. 2015. Influence of cultivar and ripeness stage at the time of fresh-cut processing on instrumental and sensory qualities of fresh-cut mangos. Postharvest Biol. Technol. 106: $11-20$.

Nordey, T., J. Joas, F. Davrieux, M. Chillet and M. Léchaudel. 2017. Robust NIRS models for non-destructive prediction of mango internal quality. Sci. Hortic. 216: 51-57.

Padda, M. S., C. V. T. Do Amarante, R. M. Garcia, D. C. 
Slaughter and E. J. Mitcham. 2011. Methods to analyze physico-chemical changes during mango ripening: A multivariate approach. Postharvest Biol. Technol. 62: 267-274.

Phetpan, K., V. Udompetaikul and P. Sirisomboon. 2018. An online visible and near-infrared spectroscopic technique for the real-time evaluation of the soluble solids content of sugarcane billets on an elevator conveyor. Comput. Electron. Agr. 154: 460-466.

Rungpichayapichet, P., B. Mahayothee, M. Nagle, P. Khuwijitjaru and J. Müller. 2016. Robust NIRS models for nondestructive prediction of postharvest fruit ripeness and quality in mango. Postharvest Biol. Technol. 111: 31-40.

Salguero-Chaparro, L., V. Baeten, O. Abbas and F. P. Rodriguez. 2012. Online analysis of intact olive fruits by Vis-NIR Spectroscopy: Optimization of the acquisition parameters. J. Food Eng. 112: 152-157.

Salguero-Chaparro, L., V. Baeten, J. A. Fernández-Pierna and F. Peña-Rodríguez. 2013. Near infrared spectroscopy (NIRS) for online determination of quality parameters in intact olives. Food Chem. 139: 1121-1126.

Schmilovitch, Z., A. Mizrach, A. Hoffman, H. Egozi and Y. Fuchs. 2000. Determination of Mango physiological indices by near-infrared spectrometry. Postharvest Biol. Technol. 19: 245-252.

Seifert, B., M. Pflanz and M. Zude. 2014. Spectral shift as ad- vanced index for fruit chlorophyll breakdown. Food Bioprocess Tech. 7: 2050-2059.

Sivakumar, D., Y. Jiang and E. M. Yahia. 2011. Maintaining mango (Mangifera indica L.) fruit quality during the export chain. Food Res. Int. 44: 1254-1263.

Slaughter, D. C. 2009. Nondestructive Maturity Assessment Methods for Mango: A Review of Literature and Identification of Future Research Needs. University of California, Davis, 1-18.

Subedi, P. P., K. B. Walsh and G. Owens. 2007. Prediction of mango eating quality at harvest using short-wave near infrared spectrometry. Postharvest Biol. Technol. 43: 326-334.

Tian, H. Q., Y. B. Ying, H. S. Lu, X. P. Fu and H. Y. Yu. 2007. Measurement of soluble solids content in watermelon by Vis/NIR diffuse transmittance technique. Journal of Zhejiang University Science B. 8: 105-110.

Ueda, M., K. Sasaki, N. Utsunomiya, K. Inaba and Y. Shimabayashi. 2000. Changes in physical and chemical properties during maturation of mango fruit (Mangifera indica L. "Irwin") cultured in a plastic greenhouse. Food Sci. Technol. Res. 6: 299-305.

Wang, W. and J. Paliwal. 2007. Near-infrared spectroscopy and imaging in food quality and safety. Sens. Instrum. Food Qual. Saf. 1: 193-207. 